\title{
Six-Degree-of-Freedom Simulation of Hypersonic Vehicles
}

\author{
Scott G. V. Frendreis * \\ Torstens Skujins ${ }^{\dagger}$ \\ Carlos E. S. Cesnik $\ddagger$
}

\begin{abstract}
A three-dimensional, six-degree-of-freedom hypersonic vehicle model is being developed that more accurately characterizes vehicle dynamics for control studies. The main focus areas for this paper are the development of the rigid body dynamics equations of motion, the parametrization of the vehicle geometry, the formulation of the three-dimensional aerodynamic loads, and the adaptation of an existing 1D propulsion model. The aerodynamic loads are calculated by using or combining two-dimensional shock/expansion theory and the Taylor-Maccoll equations for conical flow. Comparisons with computational results show good agreement of resultant force and moment for the top and bottom vehicle surfaces and for most trials for the side surfaces. Using these models, the vehicle is trimmed for steady cruise conditions, and its flight dynamics are linearized about that state. This analysis provides information regarding the stability and controllability of a generic hypersonic vehicle in three-dimensional flight.
\end{abstract}

\section{Introduction}

Maintaining controlled flight of the vehicle is among the greatest challenges facing the development of hypersonic vehicles. Complex interactions among the airframe, propulsion system, vehicle aerodynamics, and other aspects prevent each vehicle system from being developed independently. Because of this, computational vehicle simulations are a vital tool in the designing of hypersonic vehicles.

Bolender and Doman ${ }^{1}$ have developed a two-dimensional flight dynamics vehicle model used to evaluate control algorithms, shown in Fig. 1, which was further extended by Oppenheimer et al. ${ }^{2}$ Pressures and other flow properties over each surface are calculated using two-dimensional shock/expansion theory. Flexible effects $^{2}$ and control surface interactions ${ }^{3}$ have been added recently. The formulation has the capability to perform longitudinal flight simulations over a variety of vehicle trajectories in a computationally effective manner. However, the accuracy of a two-dimensional model is inherently limited due to the fact that it cannot capture three-dimensional phenomena encountered in actuality. Other longitudinal hypersonic vehicle models exist: Chavez and Schmidt ${ }^{4}$ also developed a flexible two-dimensional model using Newtonian aerodynamics and a one-dimensional propulsion model; Mirmirani et al. ${ }^{5}$ investigated the merging of experimental and computational data to generate control algorithms for hypersonic vehicles.

The overall goal of this hypersonic vehicle modeling effort is to create a three-dimensional flight simulation framework that includes coupled inertial, aerodynamic, propulsive, elastic, and thermal effects. The first step in the development of this model is to investigate three-dimensional aerodynamic effects and rigid body dynamics. This paper introduces a three-dimensional hypersonic vehicle formulation including the six-degree-of-freedom rigid body flight dynamics, an analytical method for finding the aerodynamic loads, and an existing 1D propulsion model. These are done based on a parameterized representation of the vehicle. Knowledge of the aerodynamic loads and propulsion system will be used to determine the total forces and moments and allow the performance and controllability of a vehicle to be studied.

\footnotetext{
*Graduate Research Assistant, University of Michigan, Email: scottgvf@umich.edu, Member AIAA.

${ }^{\dagger}$ Graduate Research Assistant, University of Michigan, Email: tskujins@umich.edu, Member AIAA.

${ }^{\ddagger}$ Professor, Department of Aerospace Engineering, University of Michigan, Email: cesnik@umich.edu, 1320 Beal Avenue, 3024 FXB, Ann Arbor, MI 48109-2140, Ph. (734) 764-3397, Fax: (734) 764-0578, AIAA Associate Fellow.
} 


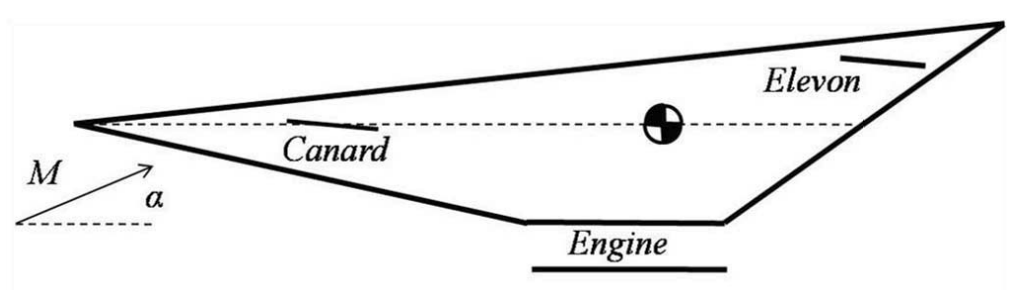

Figure 1. 2D hypersonic vehicle model $^{1}$

\section{Three-Dimensional Vehicle Simulation Framework}

The proposed 3D hypersonic vehicle simulation framework is outlined in Fig. 2. For this paper, only part of the envisioned framework has been implemented; the included simulation components are highlighted in grey. The goal of this framework is to characterize the combined longitudinal and lateral flight dynamics of hypersonic vehicles in free flight.

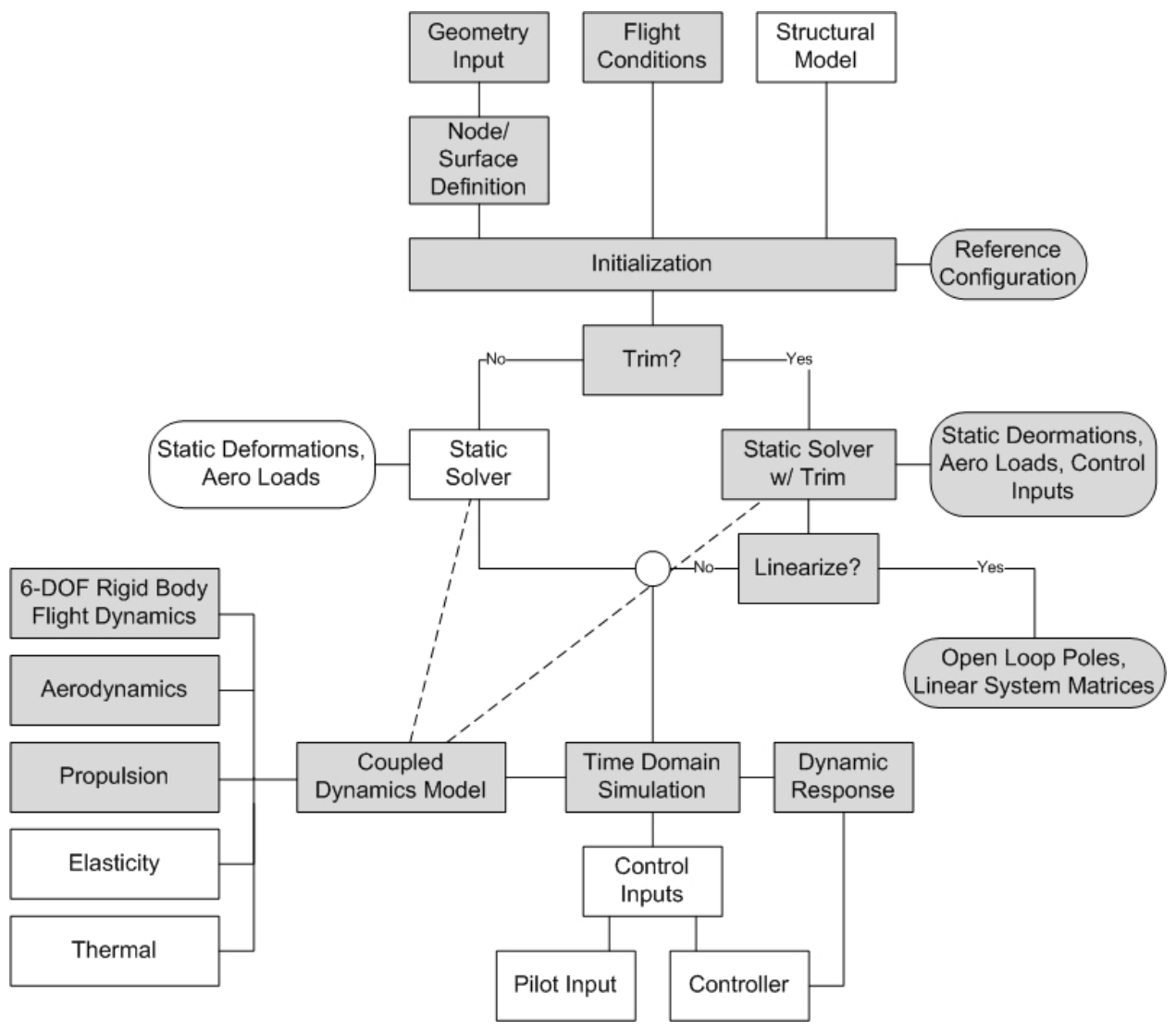

Figure 2. Proposed simulation framework

The first step of the process is to determine the equations of motion of the aircraft. Translational and rotational motions are both expressed in body fixed coordinates, and the attitude is expressed by three Euler angles. Second, the external forces and moments of the vehicle are expressed as functions of the vehicle's state. External forces will come in one of three forms: gravitation, aerodynamic, and propulsive. While gravitational force is simple to determine, the aerodynamic and propulsive effects require high fidelity modeling to accurately determine them. As a result, much of the focus of this study is on the development of satisfactory simplified models for the hypersonic aerodynamics and propulsion of the aircraft. For example, 
a full three-dimensional computational fluid dynamics (CFD) simulation would provide the desired accuracy. However, it would be much too computationally expensive to use in a time domain simulation. Therefore, it is necessary to determine a compromise between "fast" fundamental models and "slow" high-fidelity models that optimizes accuracy and computational speed. For the propulsion model, a one-dimensional representation of a scramjet is used in this first realization of the simulation framework. A three-dimensional engine is being developed by Torrez et al. ${ }^{6,7}$ and will be used in the future. A scramjet operates by slowing the incoming air to low supersonic speeds with a diffuser, adding heat to the flow, and then accelerating the air through a supersonic nozzle. By maintaining supersonic flow speeds throughout the engine, the scramjet can produce the necessary thrust to maintain hypersonic flight.

\section{A. Vehicle Geometry}

To perform six rigid body degree-of-freedom flight simulations, it is desirable to first create a generic, parameterized 3D vehicle geometry, as shown in Figure 3. It is assumed that the fuselage and cowling consist of a series of convex polygonal surfaces. Additionally, the vehicle has up to six control surfaces, which are modeled as flat trapezoidal plates.

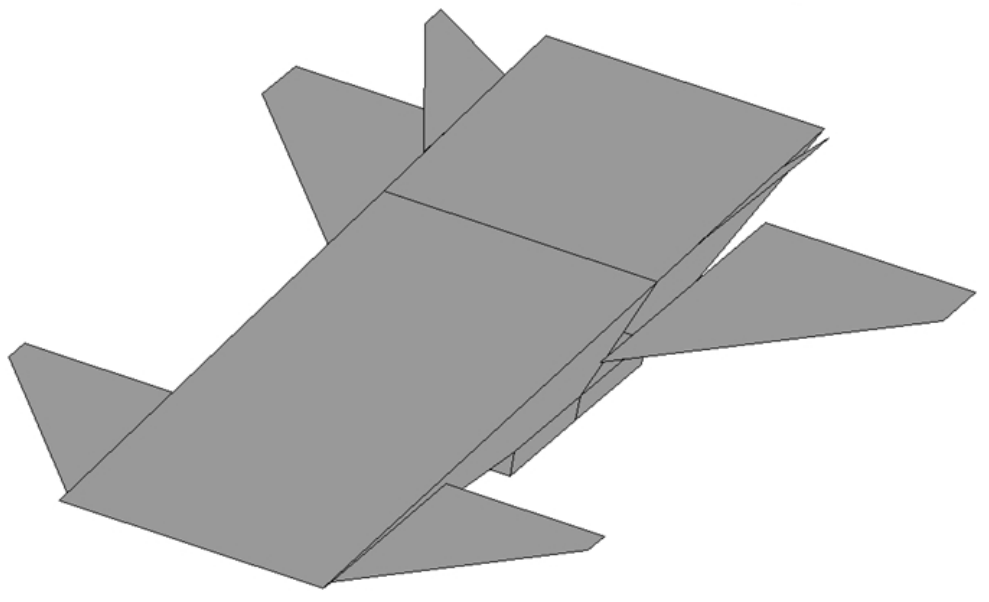

Figure 3. Generic hypersonic vehicle model

The first step of this task is to define a set of geometric parameters. Next, these parameters are used to calculate a series of 3D nodes that define the corners of each polygonal surface. Finally, the 3D nodes are used to calculate important geometric information about the surfaces (surface area, unit normal, etc.).

\section{Geometric Parameters}

The geometric parameters of the vehicle fall into one of two categories: fuselage parameters and control surface parameters. The user must define 11 parameters for the fuselage and four parameters per included control surface. These parameters allow for a range of configurations that may be used in both flight stability investigations and vehicle design.

Figure 4 shows a side view and a front view of a generic hypersonic vehicle fuselage, along with the parameters used to define the geometry. The control surfaces are omitted for clarity. The parameters are also listed and described in more detail in Table 1. Additionally, the table lists the values of these parameters used in numerical studies discussed in subsequent sections.

The vehicle may have up to six control surfaces (two canards, two elevons, and two rudders). While each of these pairs of surfaces may vary in appearance, the same set of parameters are used to define them. It is assumed that each surface is a flat trapezoidal plate, where the root and tip chord directions are parallel to each other. A generic control surface is presented in Figure 5 with the four parameters used to define it. These parameters are also listed in greater detail in Table 2. 

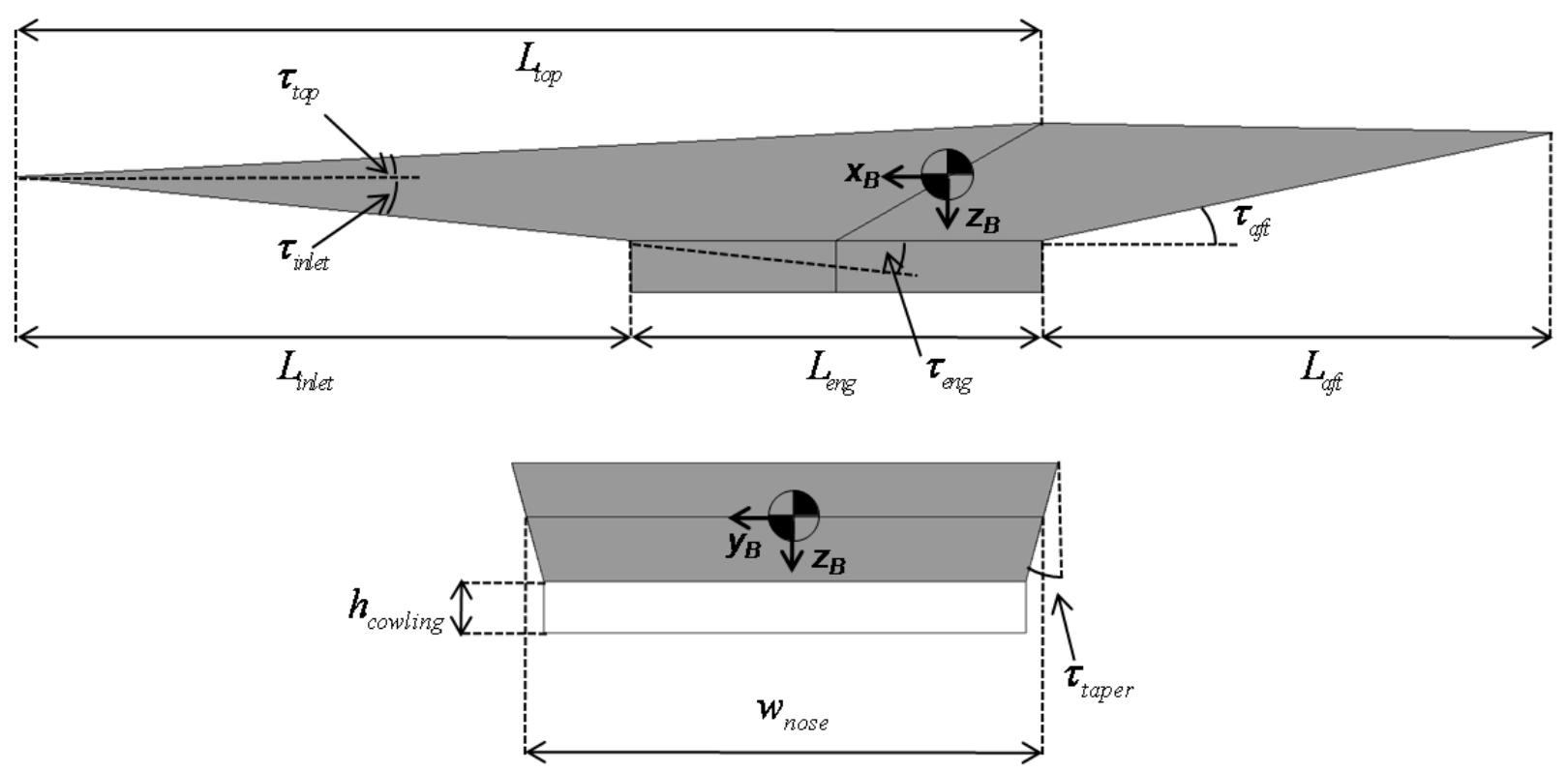

Figure 4. Fuselage Geometric Parameters

Table 1. Fuselage Geometric Parameters

\begin{tabular}{ccc}
\hline \hline Parameter & Description & Value for Numerical Studies \\
\hline$L_{\text {inlet }}$ & Inlet length & $12 \mathrm{~m}$ \\
$\tau_{\text {inlet }}$ & Inlet turn angle & $6^{\circ}$ \\
$L_{\text {eng }}$ & Engine/cowling length & $8 \mathrm{~m}$ \\
$\tau_{\text {eng }}$ & Inlet to engine turn angle & $6^{\circ}$ \\
$L_{\text {aft }}$ & Lower aftbody length & $10 \mathrm{~m}$ \\
$\tau_{\text {aft }}$ & Engine to lower aftbody turn angle & $15.82^{\circ}$ \\
$L_{\text {top }}$ & Upper forebody length & $20 \mathrm{~m}$ \\
$\tau_{\text {top }}$ & Upper forebody turn angle & $3^{\circ}$ \\
$w_{\text {nose }}$ & Width at nose & $10 \mathrm{~m}$ \\
$\tau_{\text {taper }}$ & Side taper angle & $0^{\circ}$ \\
$h_{\text {cowling }}$ & Cowling height & $1 \mathrm{~m}$ \\
\hline \hline
\end{tabular}

Table 2. Control Surface Geometric Parameters

\begin{tabular}{cc}
\hline \hline Parameter & Description \\
\hline$c_{\text {root }}$ & Chord at control surface root \\
$c_{\text {tip }}$ & Chord at control surface tip \\
$s$ & Control surface span \\
$\tau_{\text {sweep }}$ & Leading edge sweep angle \\
\hline \hline
\end{tabular}




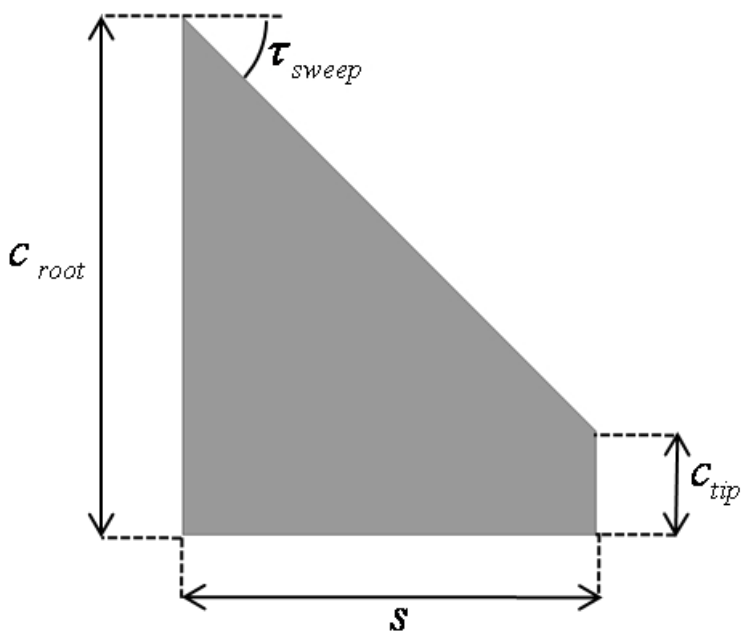

Figure 5. Control Surface Geometric Parameters

\section{Surface Definition}

Once the geometric parameters are set, they are used to define the individual surfaces that compose the vehicle exterior. This is achieved by first calculating the locations of the corners of each surface and tabulating them as a list of nodes. These nodes are expressed in a body-fixed reference frame, where the origin is placed at the center of mass, as shown in Fig. 4. Then, each surface is represented as an ordered set of node locations. Since most of the vehicle's surfaces are quadrilaterals (e.g., the fuselage top and bottom, the cowling surfaces, and the control surfaces), they are always expressed as a set of four nodes. If a surface has more than four edges it is split into a series of convex quadrilaterals, like the fuselage side in Figure 4.

The final step in defining the geometry is calculating the area, centroid, and unit normal vector of each surface of the vehicle. This information is particularly useful for determining the aerodynamic force and moments on the vehicle. As an example, consider a surface with a constant pressure distribution. In the context of hypersonic aerodynamics, this constant pressure distribution may be predicted using Newtonian aerodynamics or $2 \mathrm{D}$ shock-expansion theory. The resultant force due the pressure is equal to the product of the area and pressure, acts at the centroid of the surface, and is oriented parallel to the unit normal vector. In this manner, the geometric analysis of the vehicle surfaces is a necessary component in characterizing the overall vehicle flight dynamics.

\section{B. 6-DOF Flight Dynamics Equations of Motion}

The six degree-of-freedom equations of motion are derived using a Newtonian approach with the inertial, flat-earth assumption. Both translational and rotational velocities are expressed in a body-fixed reference frame, whose origin is located at the aircraft's center of mass and its orientation along the vehicle's principal axes. This body-fixed frame is shown in Fig. 4. The translational and rotational accelerations are associated with the resulting forces and moments as

$$
\begin{gathered}
m \dot{\vec{V}}_{B}+m \vec{\omega}_{B} \times \vec{V}_{B}=\vec{F}_{B} \\
\mathbf{I} \cdot \dot{\vec{\omega}}_{B}+\vec{\omega}_{B} \times\left(\mathbf{I} \cdot \vec{\omega}_{B}\right)=\vec{M}_{B}
\end{gathered}
$$

where $m$ is the vehicle mass, $\overrightarrow{V_{B}}$ is the body linear velocity vector, $\overrightarrow{\omega_{B}}$ is the body rotational velocity vector, and $\mathbf{I}$ is the inertia matrix with respect to the vehicle's center of mass. The external forces and moments come from the gravitational, aerodynamic, and propulsive sources. For the remainder of this paper, it will be convenient to express these equations in terms of their $\mathrm{x}, \mathrm{y}$, and $\mathrm{z}$ components. Stenge ${ }^{8}$ writes Eq. 1 as 


$$
\begin{aligned}
& \dot{u}=(r v-q w)+\frac{F_{B, x}}{m} \\
& \dot{v}=(p w-r u)+\frac{F_{B, y}}{m} \\
& \dot{w}=(q u-p v)+\frac{F_{B, z}}{m}
\end{aligned}
$$

and Eq. 2 as

$$
\begin{aligned}
& \dot{p}=\frac{\left(I_{y y}-I_{z z}\right) q r+M_{B, x}}{I_{x x}} \\
& \dot{q}=\frac{\left(I_{z z}-I_{x x}\right) r p+M_{B, y}}{I_{y y}} \\
& \dot{p}=\frac{\left(I_{x x}-I_{y y}\right) p q+M_{B, z}}{I_{z z}}
\end{aligned}
$$

where $u, v$, and $w$ are the translational velocity components of $\vec{V}_{B} ; p, q$, and $r$ are the angular velocity components of $\vec{\omega}_{B}$; and $I_{x x}, I_{y y}$, and $I_{z z}$ are the principal mass moments of inertia associated with $\mathbf{I}$. All these quantities are expressed with respect to the body fixed reference system.

The attitude of the aircraft is expressed in terms of three Euler angles. The Euler angles will be sufficient for this problem because it is assumed that aircraft pitch will remain below $\pm 90^{\circ}$. The Euler angle kinematics are determined from

$$
\left\{\begin{array}{c}
\dot{\phi} \\
\dot{\theta} \\
\dot{\psi}
\end{array}\right\}=\left[\begin{array}{ccc}
1 & \sin \phi \tan \theta & \cos \phi \tan \theta \\
0 & \cos \phi & -\sin \phi \\
0 & \frac{\sin \phi}{\cos \theta} & \frac{\cos \phi}{\cos \theta}
\end{array}\right]\left\{\begin{array}{l}
p \\
q \\
r
\end{array}\right\}
$$

where $\phi, \theta$, and $\psi$ are the roll, pitch and yaw Euler angles, respectively.

The position of the aircraft is given in terms of Earth-fixed inertial coordinates. The inertial position kinematics are calculated with

$$
\left\{\begin{array}{c}
\dot{x}_{E} \\
\dot{y}_{E} \\
\dot{z}_{E}
\end{array}\right\}=T_{E B}(\phi, \theta, \psi)\left\{\begin{array}{c}
u \\
v \\
w
\end{array}\right\}
$$

where $T_{E B}$ is the body frame-to-Earth frame transformation matrix.

\section{Aerodynamic Loads}

In order to determine the forces and moments on the vehicle due to aerodynamic loads, an analytical formulation of the pressure distribution over the vehicle is devised. The goal of the formulation here is to calculate the pressures on all surfaces of the vehicle with minimal computational cost. There have been two groups of work that inspired the development presented here. First, the work of Starkey and Lewis ${ }^{9}$ has presented a method for predicting the pressure on the surface of irregularly shaped sharp-nosed geometries in hypersonic flow by blending 2D shock/expansion and conical flow theories based on the local geometric properties at a certain location. Nastase et al. subdivided the flow in different regions depending on the influence of the incoming flow. They developed methods for finding the pressure distribution over rectangular flat plates ${ }^{10}$ and delta wings ${ }^{11}$ based on the solution of potential flow differential equations. In what follows, the relevant theories to this formulation are summarized and the application of the individual results are described for the different surfaces of the vehicle, that is, front (top and bottom) and sides.

\section{Relevant Supersonic Flow Theories}

The two formulations that will provide key information for the construction of the 3D hypersonic flow properties on finite flat plates are the 2D shock/expansion and Taylor-Maccoll equations. In the twodimensional vehicle representation, shock/expansion theory was used to find the pressures over each of the vehicle surfaces (represented by lines in this 2D case). The implementation of these shock and expansion equations is outlined in Ref. [3].

Supersonic flow around an axisymmetric body of revolution is governed by the Taylor-Maccoll equations, which have as independent variables the post-shock flow velocity $V^{\prime}$ and cone angle $\theta .{ }^{9}$ 


$$
\frac{\gamma-1}{2}\left[1-V_{r}^{\prime 2}-\frac{d V_{r}^{\prime}}{d \theta}\right]\left[2 V_{r}^{\prime}+\frac{d V_{r}^{\prime}}{d \theta} \cot \theta+\frac{d^{2} V_{r}^{\prime}}{d \theta^{2}}\right]-\frac{d V_{r}^{\prime}}{d \theta}\left[V_{r}^{\prime} \frac{d V_{r}^{\prime}}{d \theta}+\frac{d V_{r}^{\prime}}{d \theta} \frac{d^{2} V_{r}^{\prime}}{d \theta^{2}}\right]=0
$$

In Eq. 7,

$$
\begin{aligned}
V^{\prime} & =\left[\frac{2}{(\gamma-1) M^{2}}+1\right]^{-\frac{1}{2}} \\
V_{\theta}^{\prime} & =\frac{d V_{r}^{\prime}}{d \theta} \\
\frac{d V_{\theta}^{\prime}}{d \theta} & =\frac{d^{2} V_{r}^{\prime}}{d \theta^{2}}
\end{aligned}
$$

The solutions to these equations were obtained using look-up tables ${ }^{12}$ in order to incur the least amount of computational cost.

\section{Front Surface}

The surfaces on the top and bottom of the vehicle are considered first. The bottom front and bottom middle surfaces will be the focus in the subsequent discussion. In reality on the hypersonic vehicle, the engine and cowling will be located on the bottom middle surface, as shown in Fig. 4. However, the formulation is valid for any situation where the flow passes through an expansion fan or shock from one surface to the next as it travels downstream, so the presence of the engine is not relevant for the validation of this model.

Consider a rectangular flat plat, as shown in Fig. 6. Assume that the plate is oriented at an angle with respect to the freestream flow direction such that a shock forms at the leading edge. If the plate was infinitely wide, then no edge effects would be present. However, since the plate is of finite width, the flow near the edges will be affected by the edges. In the middle of the surface, where no edge effects are present, the flow behind the shock is effectively 2D. At some point along the span, edge effects will begin to be felt. The basis of the formulation for this surface is to determine the regions in which the edge effects are significant. Region A in Fig. 6 corresponds to the locations outside of the edge-affected areas on the surface. The two-dimensional shock/expansion equations will be used to determine the flow properties there.

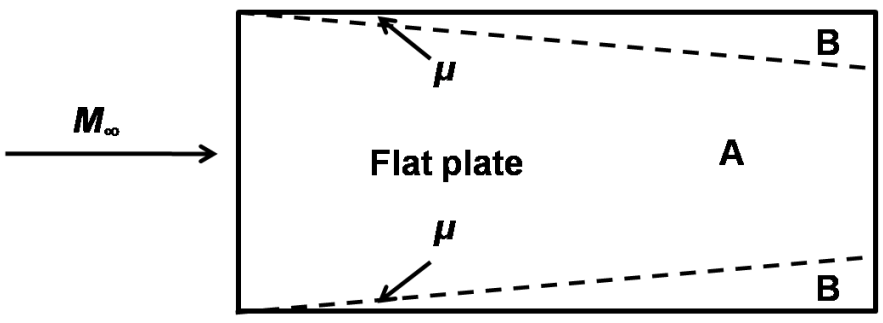

Figure 6. Flat plate edge-affected regions

Region B is the area where the edge effects will be significant. The boundaries between regions A and $\mathrm{B}$ is determined using the Mach angle $\mu$. In supersonic flow, the Mach angle is the maximum angle with respect to the flow at which information from a disturbance can propagate downstream flow, ${ }^{10}$ defined as:

$$
\mu=\sin ^{-1}\left(\frac{1}{M}\right)
$$

The corners of the surface can be thought of as disturbances in the flow. Thus, the information from the corners can only propagate downstream at angle $\mu$. Locations outside of the Mach angle are not affected by the edges, so this angle defines the boundary between regions A and B. To find the pressure on the longitudinal edges of the plates, conical flow is used. This approximation treats the sides as if they form a cone with the cone angle equaling the bottom front surface angle $\tau_{\text {lower }}$ (or top front angle $\tau_{\text {upper }}$ for the top surface), as shown in Fig. 4. Then, the Taylor-Maccoll equations are used with this cone angle and freestream Mach number as inputs. 
To account for the pressures in the edge-affected regions, consider Fig. 7, which shows a CFD solution for the spanwise pressure distribution at a location halfway down the bottom front surface. The pressure in the middle of the surface is constant, corresponding to Region A in Fig. 6. In the edge-affected areas, the pressure decreases in a nearly linear manner to the boundary pressure. Thus, to find the pressures in the edge-affected regions, the pressure will be linearly interpolated from the boundary pressure to the $2 \mathrm{D}$ pressure from the middle of the surface.

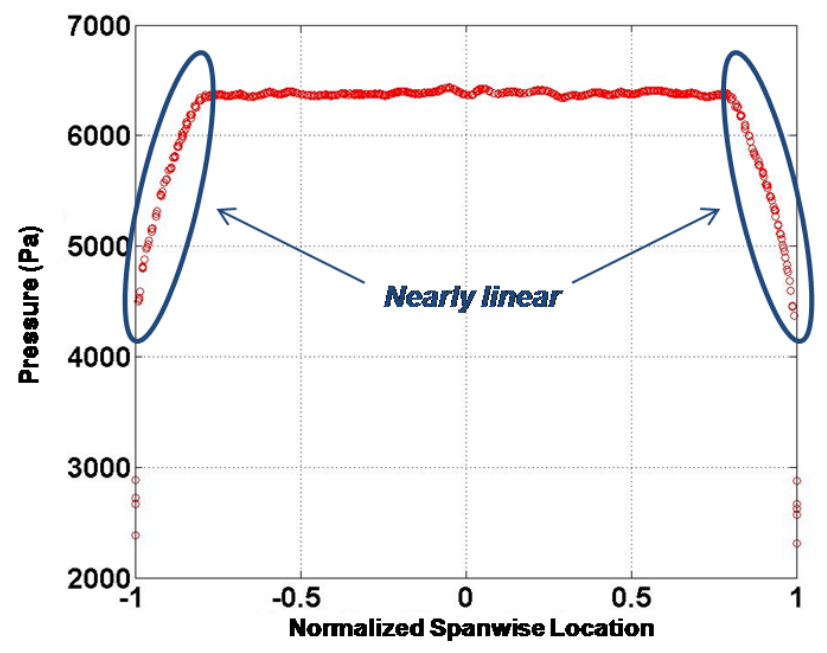

Figure 7. CFD spanwise pressure distribution, $M=8, h=85,000 \mathrm{ft}, \alpha=\beta=0^{\circ}$

The results obtained from the front surface formulation have been compared with Euler CFD solutions. All CFD runs were conducted using Metacomp's CFD++ Version 6.5.1. ${ }^{13}$ Unstructured grids were generated using both ANSYS ICEM CFD ${ }^{14}$ and Fluent's Gambit, ${ }^{15}$ depending on the specific case. For the front and middle surface tests, a grid of just over 1.6 million cells was used. A grid with approximately four times the number of cells changed the resultant force by under $1 \%$, showing that the solution is independent of the grid. Note that, for these trials, the bottom front surface was made to be rectangular, though it could have been made any trapezoidal shape. The trial parameters are displayed in Table 3 ; note that $\psi_{F}$ is the front surface flow turning angle due to both flow conditions and vehicle geometry. In this particular case, the wedge angle is $6^{\circ}$.

Table 3. CFD trial parameters (85,000 ft level flight)

\begin{tabular}{ccccc}
\hline \hline Trial & $M$ & $\alpha$ & $\beta$ & $\psi_{F}$ \\
\hline 1 & 8 & 0 & 0 & $6.00^{\circ}$ \\
2 & 8 & -3 & 0 & $3.00^{\circ}$ \\
3 & 8 & 0 & 3 & $5.99^{\circ}$ \\
4 & 8 & 3 & 3 & $8.98^{\circ}$ \\
5 & 8 & 10 & 0 & $16.0^{\circ}$ \\
6 & 6 & 0 & 0 & $6.00^{\circ}$ \\
7 & 10 & 0 & 0 & $6.00^{\circ}$ \\
\hline \hline
\end{tabular}

The resultant force and moment on the surface are shown in Tables 4 . In addition to the values calculated using the front surface formulation, the forces and moments calculated using only the 2D shock/expansion values have been included as well, labelled as "2D." The percent errors are with respect to the CFD solution.

The front surface formulation agrees well with the CFD results, differing by a maximum of $1.66 \%$ in force and $1.63 \%$ in moment in the trials tested. The strictly $2 \mathrm{D}$ formulation has a higher maximum error of 
Table 4. Front surface percent error

\begin{tabular}{|c|c|c|c|c|}
\hline \multirow[t]{2}{*}{ Trial } & \multicolumn{2}{|l|}{ Force } & \multicolumn{2}{|c|}{ Moment } \\
\hline & Formulation & $2 \mathrm{D}$ & Formulation & $2 \mathrm{D}$ \\
\hline 1 & 1.07 & 3.64 & 1.28 & 4.35 \\
\hline 2 & 0.28 & 2.24 & 0.36 & 2.93 \\
\hline 3 & 0.65 & 3.20 & 0.81 & 3.87 \\
\hline 4 & 1.66 & 4.20 & 1.63 & 4.92 \\
\hline 5 & 3.30 & 5.84 & 3.70 & 7.06 \\
\hline 6 & 0.74 & 3.95 & 1.08 & 5.11 \\
\hline 7 & 1.49 & 3.60 & 1.60 & 4.10 \\
\hline $\begin{array}{c}\text { Average } \\
\text { Improvement }\end{array}$ & \multicolumn{2}{|l|}{2.49} & \multicolumn{2}{|l|}{3.09} \\
\hline
\end{tabular}

$4.20 \%$ in force and $5.11 \%$ in moment. Figures 8 and 9 show more qualitative comparisons of the pressure distributions. Fig. 8 shows the spanwise pressure distribution at the trailing edge of the bottom front surface for two separate cases. Fig. 9 shows the pressure contour plot of the bottom front surface obtained from the proposed formulation as well as CFD solution. These two figures show that the zone of influence of the edge effects has been captured accurately by the formulation; also, the pressures agree closely across the span of the surface. Furthermore, to investigate the accuracy of the proposed formulation on an expansion fan surface, a case was run with $\alpha=10^{\circ}$ and $\beta=3^{\circ}$, and, for the top front surface, $\psi_{F}=-6.99^{\circ}$. The formulation's prediction of resultant force differed with the CFD's prediction by under $1 \%$.

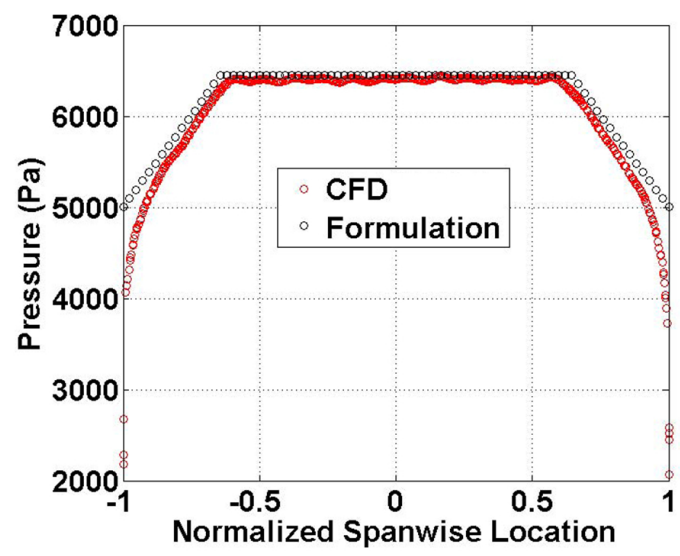

(a) $\alpha=\beta=0^{\circ}$

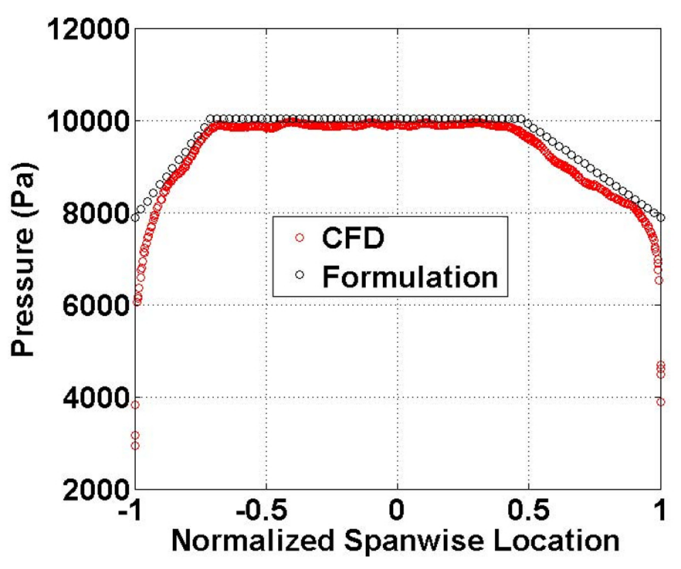

(b) $\alpha=\beta=3^{\circ}$

Figure 8. Spanwise pressure distribution for $M=8$ at the trailing edge of the bottom front surface

\section{Downstream Surfaces}

The pressure distribution on subsequent downstream surfaces is found in much the same way. The 2D pressures from the center of the surface and the boundary conditions on the edge of the surface are passed through an expansion fan. These new values are used for the downstream surface, and the edge-affected region pressures are found again by linear interpolation. Fig. 10 shows the regions on two subsequent surfaces where the $2 \mathrm{D}$ shock/expansion values are used as well as the regions of linear interpolation. Note that an expansion fan occurs at the boundary between the surfaces. 


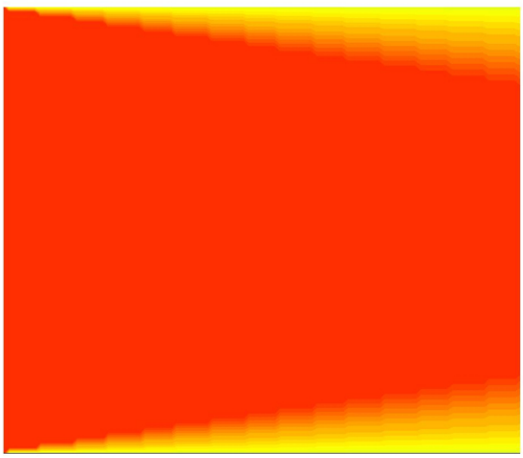

(a) Formulation

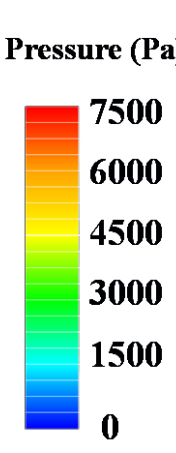

(b) $\mathrm{CFD}$

Figure 9. Front surface pressure contour plots, $M=8, \alpha=\beta=0^{\circ}$

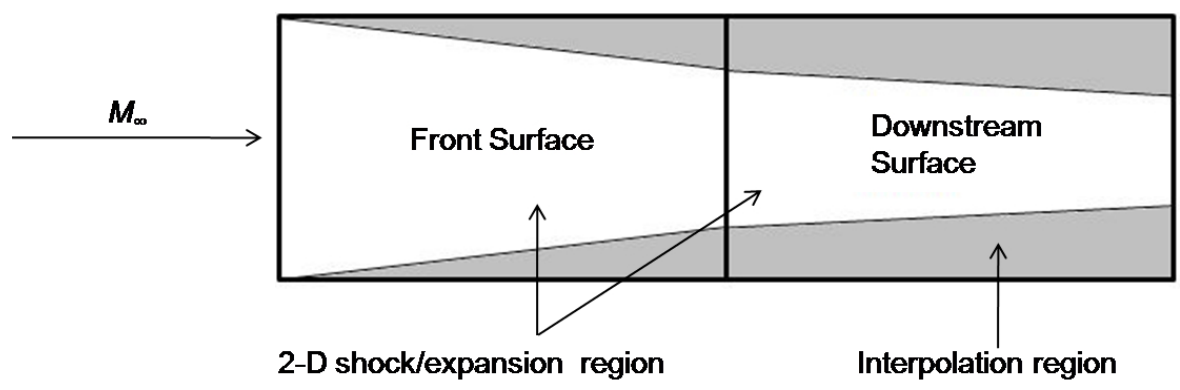

Figure 10. Surface Formulation Diagram

Table 5 shows the force and moment results for the bottom middle surface; the trials have the same parameters as in Table 3.

The errors from the formulation remain approximately the same as for the front surface, while the strictly $2 \mathrm{D}$ errors increased to over $5 \%$.

Fig. 11 shows the spanwise pressure distribution at the trailing edge of the bottom middle surface, while Fig. 12 compares the CFD and formulation pressure contours. As in the front surface, the zone of influence of edge effects is captured well. However, additional edge effects are present on both sides of Fig. 11(a) and on the right side of Fig. 11(b) (note that, in that figure, the sideslip component of the flow is traveling from right to left). After decreasing from the center 2D pressure, the pressure rises just before the boundary, a phenomenon not captured in the formulation. However, as shown in Table 5, this does not lead to significant error in the calculated forces and moments. Since the goal of this research is to devise a simplified aerodynamic model as discussed before, these effects will be neglected.

\section{Side Surfaces}

To find the pressures on the side surfaces, the angle of incidence $\psi_{s}$ between the freestream flow and side surfaces, as shown in Fig. 13, is calculated. On the shock side, when $\psi_{s}$ results in the flow turning into itself (positive $\psi_{s}$ ), the Taylor-Maccoll equations are used to find the pressure on the surface. On the shadow side, when $\psi_{s}$ results in the flow turning away from itself (negative $\psi_{s}$ ), the expansion fan equation is used. Note that, if $\tau_{\text {taper }}$ in Fig. 4 is greater than $0^{\circ}$, it is possible that vehicle flight conditions will result in the flow turning into itself on each side. Then, the Taylor-Maccoll equations are used on both side surfaces with the respective values of $\psi_{s}$ on each side. 
Table 5. Middle surface percent error

\begin{tabular}{|c|c|c|c|c|}
\hline \multirow[t]{2}{*}{ Trial } & \multicolumn{2}{|l|}{ Force } & \multicolumn{2}{|c|}{ Moment } \\
\hline & Formulation & $2 \mathrm{D}$ & Formulation & $2 \mathrm{D}$ \\
\hline 1 & 1.57 & 7.32 & 1.65 & 7.38 \\
\hline 2 & -1.10 & 3.48 & -0.91 & 3.43 \\
\hline 3 & 0.30 & 5.97 & 0.35 & 6.01 \\
\hline 4 & 1.93 & 8.61 & 2.29 & 8.68 \\
\hline 5 & 4.77 & 13.2 & 4.93 & 13.5 \\
\hline 6 & 1.66 & 9.10 & 1.70 & 9.18 \\
\hline 7 & 0.47 & 5.19 & 0.59 & 5.30 \\
\hline $\begin{array}{c}\text { Average } \\
\text { Improvement }\end{array}$ & \multicolumn{2}{|l|}{5.44} & \multicolumn{2}{|l|}{5.42} \\
\hline
\end{tabular}

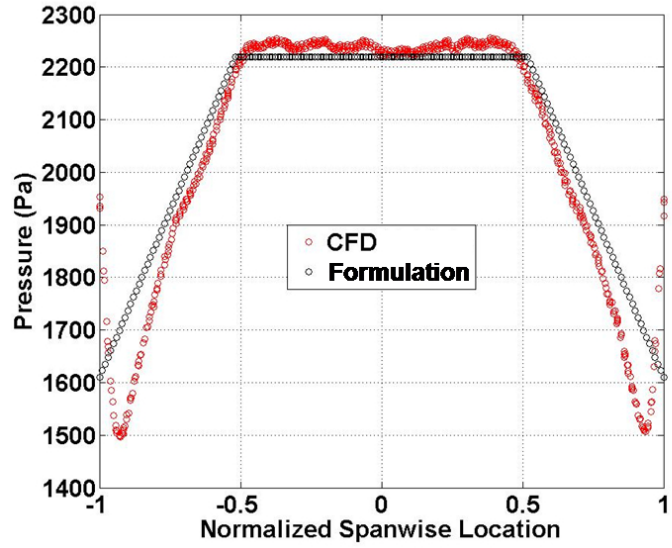

(a) $\alpha=\beta=0^{\circ}$

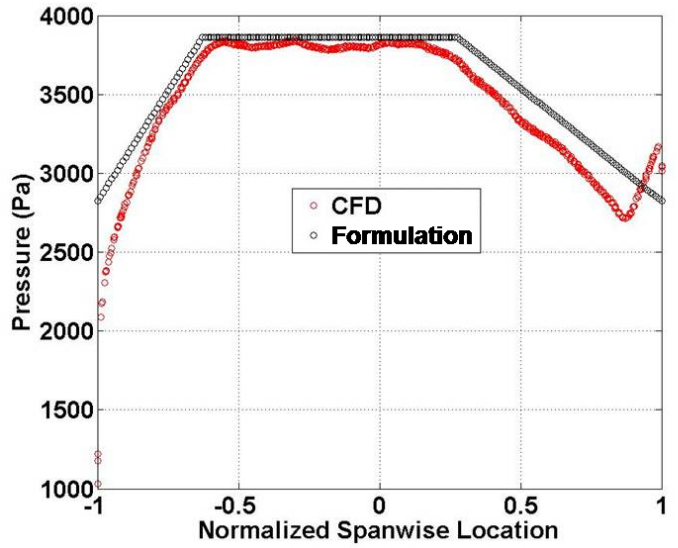

(b) $\alpha=\beta=3^{\circ}$

Figure 11. Spanwise pressure distribution for $M=8$ at the trailing edge of the bottom middle surface

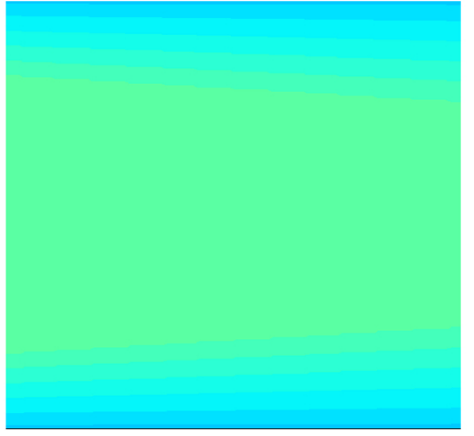

(a) Formulation

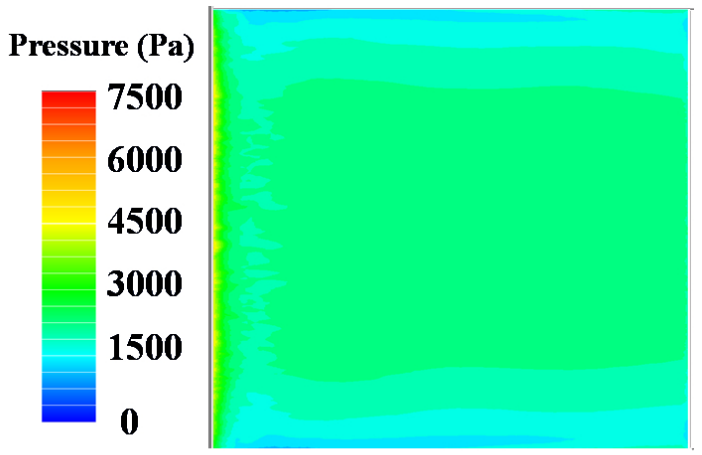

(b) CFD

Figure 12. Middle surface pressure contour plots, $M=8, \alpha=\beta=0^{\circ}$ 


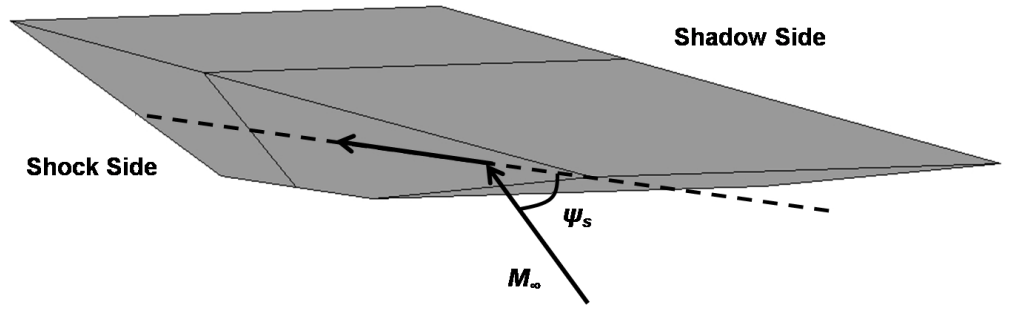

Figure 13. $\psi_{s}$ definition

To test the side surface formulation, in addition to the geometry with rectangular top and bottom surfaces described in Section II.A.1, a hypersonic vehicle geometry was constructed such that $\tau_{\text {taper }}=45^{\circ}$. This angle value was chosen because it is in the upper range of values of $\tau_{\text {taper }}$ that would be expected to be seen on a hypersonic vehicle. Thus, the two $\tau_{\text {taper }}$ values are at or near the extremes of that portion of the design space. A CFD mesh with just over 8 million grid points was used for this new configuration. A mesh with over twice as many grid points changed the resultant force on the surface by under $1 \%$, showing that the solution is independent of the grid. Tables $7(\mathrm{a})$ and $7(\mathrm{~b})$ show the percentage difference between the side surface formulation and the CFD solutions. The trials correspond to those in Tables 6(a) and 6(b). For the cases with sideslip present, results from each of the side surfaces are presented; in the trial number, $S$ stands for the shock side surface and $E$ for the shadow side, which may have either an expansion or a weaker shock depending on the sign of $\psi_{s}$, as described previously.

Table 6. CFD trial parameters $(85,000 \mathrm{ft}$ level flight)

(a) $\tau_{\text {taper }}=45^{\circ}$

\begin{tabular}{ccccc}
\hline \hline Trial & $M$ & $\alpha$ & $\beta$ & $\psi_{s}$ \\
\hline$A 1$ & 8 & 0 & 0 & 0 \\
$A 2$ & 8 & 0 & 1.5 & \pm 1.06 \\
$A 3$ & 8 & 0 & 3 & \pm 2.12 \\
$A 4$ & 8 & 3 & 0 & 2.12 \\
$A 5$ & 8 & 5 & 0 & 3.53 \\
$A 6$ & 8 & 3 & 3 & $4.24 /-0.003$ \\
$A 7$ & 8 & 5 & 3 & $5.66 / 1.41$ \\
$A 8$ & 8 & 10 & 0 & 7.05 \\
$A 9$ & 8 & 10 & 3 & $9.18 / 4.91$ \\
\hline \hline
\end{tabular}

(b) $\tau_{\text {taper }}=0^{\circ}$

\begin{tabular}{ccccc}
\multicolumn{5}{c}{$(\mathrm{b}) \tau_{\text {taper }}=0^{\circ}$} \\
\hline \hline Trial & $M$ & $\alpha$ & $\beta$ & $\psi_{s}$ \\
\hline$B 1$ & 8 & 0 & 3 & \pm 3 \\
$B 2$ & 8 & 2 & 1 & \pm 1 \\
$B 3$ & 8 & 3 & 3 & \pm 3 \\
$B 4$ & 8 & 5 & 1.5 & \pm 1.5 \\
$B 5$ & 8 & 5 & 3 & \pm 3 \\
$B 6$ & 8 & 10 & 3 & \pm 3 \\
\hline \hline
\end{tabular}

Table 7(a) shows generally good agreement between the calculated forces and moments and CFD results for the shock side, with most errors under $10 \%$ and a maximum force error of $12.9 \%$ for Trial $A 8$. The expansion side errors are larger, up to $25.5 \%$ for the force in Trial $A 7$. However, the pressure values themselves on the expansion sides are less than those on the shock sides. For example, in Trial $A 7$, the expansion side pressure from the CFD results is $13.4 \mathrm{kPa}$, while the shock side has a much higher value of $31.5 \mathrm{kPa}$. Thus, a larger percent error on the expansion side will have less effect on vehicle dynamics than a similar error on the shock side due to the absolute magnitude of the pressure values.

For the trials having $\tau_{\text {taper }}=0^{\circ}$, the errors on the shock side are generally under $10 \%$, other than Trial B4. As with the results for $\tau_{\text {taper }}=45^{\circ}$, the expansion side errors are higher. In the same way as for the $\tau_{\text {taper }}=45^{\circ}$ trials, these errors will have a relatively small effect on vehicle dynamics when compared to the shock side. Fig. 14 shows the pressure distribution at the indicated cut; note that the bottom of the vehicle corresponds to the left side of the plot. 
Table 7. Side surface percent error

(a) $\tau_{\text {taper }}=45^{\circ}$

\begin{tabular}{crrrrrr}
\hline \hline Trial & Force & Moment & & Trial & Force & Moment \\
\cline { 1 - 3 } \cline { 5 - 7 }$A 1$ & 7.04 & 7.75 & & $B 1 S$ & -8.29 & -8.34 \\
$A 2 S$ & -1.63 & -0.05 & & $B 1 E$ & 16.6 & 16.5 \\
$A 2 E$ & 7.35 & -7.06 & & $B 2 S$ & 5.36 & 2.27 \\
$A 3 S$ & -10.4 & -7.90 & & $B 2 E$ & 10.6 & 7.53 \\
$A 3 E$ & 6.29 & 5.53 & & $B 3 S$ & -0.10 & -0.06 \\
$A 4$ & 1.89 & 3.47 & & $B 3 E$ & 12.0 & 7.91 \\
$A 5$ & 5.97 & 7.83 & & $B 4 S$ & 18.5 & 17.5 \\
$A 6 S$ & -5.80 & -3.00 & & $B 4 E$ & 27.4 & 22.5 \\
$A 6 E$ & 24.1 & 25.1 & & $B 5 S$ & 5.17 & 5.22 \\
$A 7 S$ & -1.77 & 1.34 & & $B 5 E$ & 17.4 & 11.9 \\
$A 7 E$ & 25.5 & 27.1 & & $B 6 S$ & 8.41 & 8.59 \\
$A 8$ & 12.9 & 14.6 & & $B 6 E$ & 49.0 & 49.7 \\
$A 9 S$ & 7.55 & 10.7 & & & & \\
$A 9 E$ & 18.4 & 20.2 & & & & \\
\hline \hline
\end{tabular}

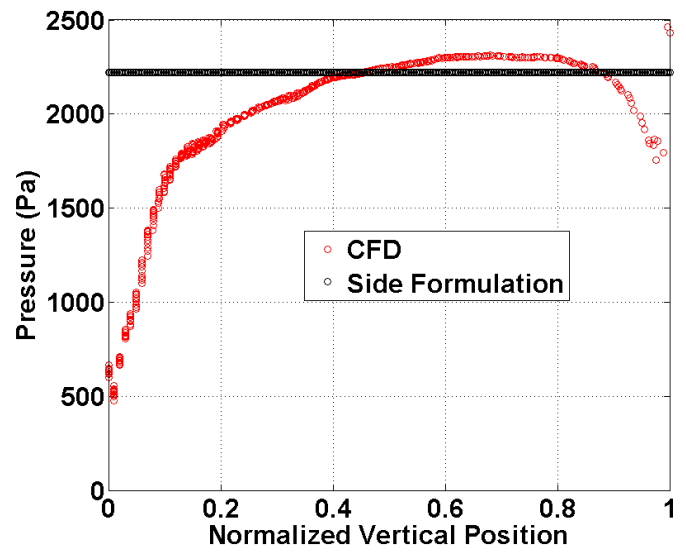

(a) Trial $A 1$

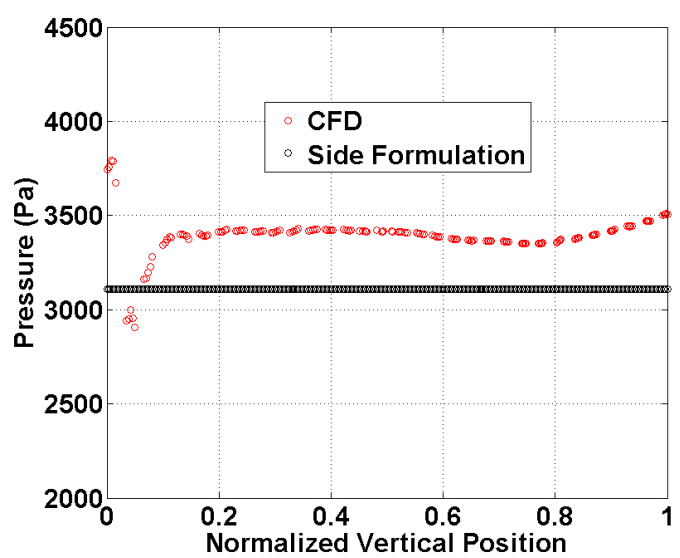

(b) Trial $B 1 S$

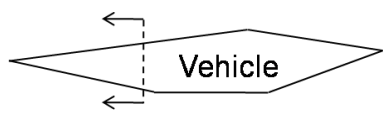

Figure 14. Side surface pressure distribution at indicated cut, $M=8$ 


\section{Propulsion}

For this simulation, the scramjet model uses one-dimensional flow equations, as developed by Bolender and Doman. ${ }^{1}$ A schematic of the scramjet is shown in Figure 15. Note that, in the equations which follow, the subscripts refer to the zones shown in the same figure.

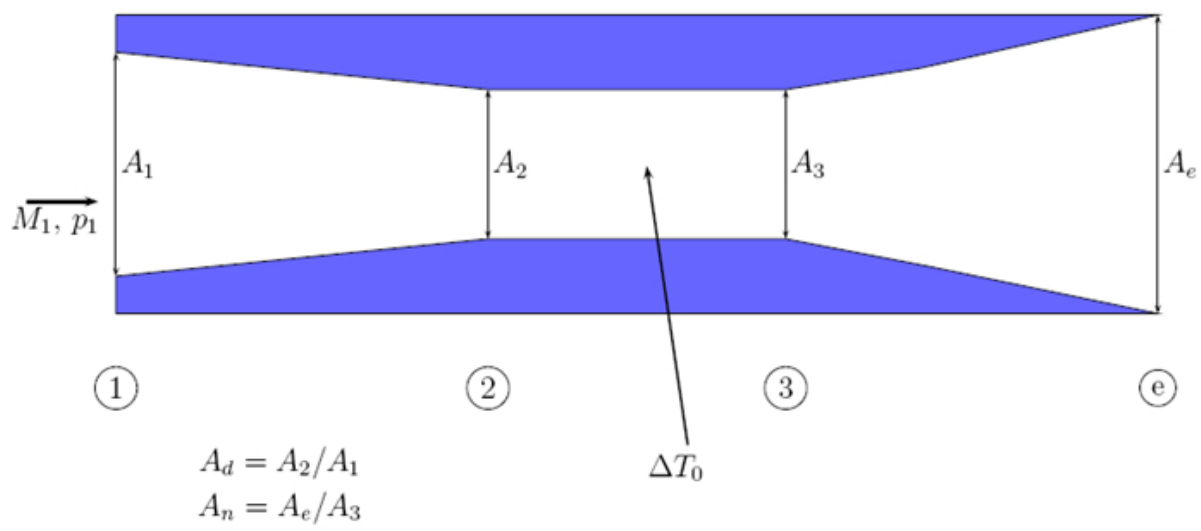

Figure 15. Scramjet diagram

It is assumed that the incoming air passes through a single oblique shock to align the flow with the engine. The first stage of the engine is an isentropic diffuser, which is represented by the isentropic mass continuity as

$$
\frac{\left[1+\frac{\gamma-1}{2} M_{2}^{2}\right]^{\frac{\gamma+1}{\gamma-1}}}{M_{2}^{2}}=A_{d}^{2} \frac{\left[1+\frac{\gamma-1}{2} M_{1}^{2}\right]^{\frac{\gamma+1}{\gamma-1}}}{M_{1}^{2}}
$$

where $M_{1}$ is the pre-diffuser Mach number, $M_{2}$ is the post-diffuser/pre-combustor Mach number, and $A_{d}$ is the diffuser area ratio. The combustor is treated as a constant area, frictionless duct with heat addition. The total temperature change in the combustor is governed by

$$
\frac{T_{03}}{T_{02}}=\frac{1+H_{f} \eta_{c} f_{s t} / c_{p} T_{02}}{1+f_{s t} \Phi}
$$

where $T_{02}$ and $T_{03}$ are the respective pre- and post-combustor total temperatures, $c_{p}$ is the specific heat capacity of air, $H_{f}$ is the lower heating value of the fuel, $\eta_{c}$ is the combustor efficiency, $f_{s t}$ is the stoichiometric fuel-to-air mass ratio, and $\Phi$ is the fuel equivalence ratio. Using the total temperature change, the postcombustor Mach number $M_{3}$ of the flow is given by

$$
\frac{M_{3}^{2}\left[1+\frac{\gamma-1}{2} M_{3}^{2}\right]}{\left(\gamma M_{3}^{2}+1\right)^{2}}=\frac{M_{2}^{2}\left(1+\frac{\gamma-1}{2} M_{2}^{2}\right)}{\left(\gamma M_{2}^{2}+1\right)^{2}}+\frac{M_{2}^{2}}{\left(\gamma M_{2}^{2}+1\right)^{2}} \frac{\left(T_{03}-T_{02}\right)}{T_{2}}
$$

Using the pre- and post-combustor Mach numbers, the post-combustor pressure and temperature are determined by the Rayleigh line relations

$$
\begin{gathered}
p_{3}=p_{2} \frac{1+\gamma M_{2}^{2}}{1+\gamma M_{3}^{2}} \\
T_{3}=\frac{M_{3}^{2}}{M_{2}^{2}} \frac{\left(1+\gamma M_{2}^{2}\right)^{2}}{\left(1+\gamma M_{1}^{2}\right)^{2}}
\end{gathered}
$$

where $p_{2}$ and $T_{2}$ are the pressure and temperature before combustion, and $p_{3}$ and $T_{3}$ are the pressure and temperature after combustion, respectively. 
The final stage of the engine is an isentropic supersonic nozzle. Since it is assumed to be isentropic, it is also governed by Eq. 10. However, $M_{1}$ is replaced by $M_{3}, M_{2}$ is replaced by the engine exit Mach number $M_{e}$, and $A_{d}$ is replaced by the nozzle area ratio, $A_{n}$.

Finally, using momentum mechanics, the thrust $\mathcal{T}$ is determined by

$$
\mathcal{T}=\dot{m}_{a}\left[(1+f) V_{e}-V_{\infty}\right]+\left(p_{e}-p_{\infty}\right) A_{e}-\left(p_{1}-p_{\infty}\right) A_{1}
$$

where $\dot{m}_{a}$ is the engine inflow mass flow rate, $f$ is the fuel-air ratio, $V_{e}$ is the flow's exit velocity, $V_{\infty}$ is the vehicle free stream velocity, $P_{e}$ is the exit pressure, $P_{\infty}$ is the free stream air pressure, $A_{e}$ is the engine exit area, and $A_{1}$ is the engine inlet area.

\section{Numerical Results from Flight Dynamics Analysis}

Using the 3D aerodynamic and propulsive models developed thus far, it is now possible to characterize the flight dynamics of the aircraft. This task first requires that the aircraft be trimmed at a desired flight condition. Then, the flight dynamics can be linearized about the trimmed state. Linearizing the flight dynamics provides valuable insight regarding the stability and controllability of the aircraft.

The first step of this analysis is to compare this model with an existing hypersonic vehicle code. For this study, and 2D hypersonic vehicle code was provided by the Air Force Research Laboratory (AFRL). ${ }^{1}$ Comparing the trim and linearization results of the 3D formulation (constrained to longitudinal flight) and the 2D AFRL one serves to verify the basic code implementation. A similar dynamic analysis is performed on the generic hypersonic vehicle configuration in $3 \mathrm{D}$ flight.

\section{A. Comparison with 2D Model}

To verify the $3 \mathrm{D}$ code, the vehicle is constrained to longitudinal flight, and its results are compared with those of the existing AFRL code. The geometric parameters of the vehicle are set to match those used by Bolender and Doman. ${ }^{1}$ The aircraft is then trimmed for Mach 8, steady-level flight at an altitude of 85,000 feet. The trimmed state and input variables used for both codes are presented in Table 8.

Table 8. Longitudinal Trim Conditions

\begin{tabular}{cc}
\hline \hline State & Value \\
\hline$V$ & $7847 \mathrm{ft} / \mathrm{s}$ \\
$h\left(-z_{E}\right)$ & $85,000 \mathrm{ft}$ \\
$\alpha$ & $1.643 \mathrm{deg}$ \\
$\theta$ & $1.643 \mathrm{deg}$ \\
$q$ & $0 \mathrm{deg} / \mathrm{s}$ \\
$\delta_{c}$ & $-7.716 \mathrm{deg}$ \\
$\delta_{e}$ & $6.185 \mathrm{deg}$ \\
$\Phi$ & 0.6298 \\
\hline \hline
\end{tabular}

The rigid body flight dynamics are then linearized about the trim states. The eigenvalues of the system using the 2D code are given in Table 9, and the eigenvalues using the $3 \mathrm{D}$ code are given in Table 10. Additionally, the eigenvectors for the system are presented in Tables 11 (2D code) and 12 (3D code).

There is strong agreement between the 2D and 3D linearization results for the short period and altitude modes. However, there is noticeable discrepancy between the real component of the phugoid mode eigenvalues $(\sim 13 \%)$. This variation causes a shift in the phugoid mode damping ratio, while minimally impacting its natural frequency. It was determined through variation of model parameters (vehicle mass, gravitational acceleration, etc.) that the real part of the phugoid eigenvalue is very sensitive to small changes in the flight dynamics model. Therefore, the discrepancy between phugoid mode eigenvalues is due to slight differences between the 2D AFRL code and the present 3D code. 
Table 9. Eigenvalues of Linearized System (2D Model)

\begin{tabular}{cccc}
\hline \hline Eigenvalue & Damping Ratio & Natural Frequency $(\mathrm{rad} / \mathrm{s})$ & Mode \\
\hline 4.70 & -1.00 & 4.70 & Short Period \\
-4.82 & 1.00 & 4.82 & Short Period \\
$-1.04 \times 10^{-5} \pm 3.92 \times 10^{-2} j$ & $2.65 \times 10^{-4}$ & $3.92 \times 10^{-2}$ & Phugoid \\
$-1.84 \times 10^{-3}$ & 1.00 & $1.84 \times 10^{-3}$ & Altitude \\
\hline \hline
\end{tabular}

Table 10. Eigenvalues of Linearized System (3D Model)

\begin{tabular}{cccc}
\hline \hline Eigenvalue & Damping Ratio & Natural Frequency $(\mathrm{rad} / \mathrm{s})$ & Mode \\
\hline 4.70 & -1.00 & 4.70 & Short Period \\
-4.82 & 1.00 & 4.82 & Short Period \\
$-9.20 \times 10^{-6} \pm 3.91 \times 10^{-2} j$ & $2.35 \times 10^{-4}$ & $3.91 \times 10^{2}$ & Phugoid \\
$-1.84 \times 10^{-3}$ & 1.00 & $1.84 \times 10^{-3}$ & Altitude \\
\hline \hline
\end{tabular}

Table 11. Eigenvectors of Linearized System (2D Model)

\begin{tabular}{ccccc}
\hline \hline State & Short Period & Short Period & Phugoid & Altitude \\
\hline$V$ & $4.75 \times 10^{-1}$ & $-4.79 \times 10^{-1}$ & $-4.06 \times 10^{-3} \pm 1.02 \times 10^{-4} j$ & $3.02 \times 10^{-1}$ \\
$h\left(-z_{E}\right)$ & $-8.74 \times 10^{-1}$ & $-8.72 \times 10^{-1}$ & 1.00 & $9.53 \times 10^{-1}$ \\
$\alpha$ & $-2.02 \times 10^{-2}$ & $-2.12 \times 10^{-2}$ & $5.73 \times 10^{-9} \pm 2.23 \times 10^{-10} j$ & $-3.36 \times 10^{-7}$ \\
$\theta$ & $-2.07 \times 10^{-2}$ & $-2.06 \times 10^{-2}$ & $4.40 \times 10^{-9} \pm 4.99 \times 10^{-6} j$ & $-5.59 \times 10^{-7}$ \\
$q$ & $-9.73 \times 10^{-2}$ & $9.96 \times 10^{-2}$ & $-1.95 \times 10^{-7} \pm 1.21 \times 10^{-10} j$ & $1.03 \times 10^{-9}$ \\
\hline \hline
\end{tabular}

Table 12. Eigenvectors of Linearized System (3D Model)

\begin{tabular}{ccccc}
\hline \hline State & Short Period & Short Period & Phugoid & Altitude \\
\hline$V$ & $4.75 \times 10^{-1}$ & $-4.79 \times 10^{-1}$ & $-4.07 \times 10^{-3} \pm 8.28 \times 10^{-5} j$ & $3.01 \times 10^{-1}$ \\
$h\left(-z_{E}\right)$ & $-8.74 \times 10^{-1}$ & $-8.71 \times 10^{-1}$ & 1.00 & $9.54 \times 10^{-1}$ \\
$\alpha$ & $-2.02 \times 10^{-2}$ & $-2.12 \times 10^{-2}$ & $6.37 \times 10^{-9} \pm 2.45 \times 10^{-10} j$ & $-3.35 \times 10^{-7}$ \\
$\theta$ & $-2.07 \times 10^{-2}$ & $-2.06 \times 10^{-2}$ & $5.12 \times 10^{-9} \pm 4.99 \times 10^{-6} j$ & $-5.58 \times 10^{-7}$ \\
$q$ & $-9.73 \times 10^{-2}$ & $9.96 \times 10^{-2}$ & $-1.95 \times 10^{-7} \pm 1.58 \times 10^{-10} j$ & $1.03 \times 10^{-9}$ \\
\hline \hline
\end{tabular}

16 of 19

American Institute of Aeronautics and Astronautics 


\section{B. 3D Analysis}

Next, the trim and linearization procedure is applied to 3D flight. The values of the vehicle geometric parameters used for this analysis are given in Table 1. Additionally, the mass properties of the vehicle, expressed in the body-fixed reference system, are given in Table 13. The center of mass of the vehicle is assumed to be at a location $60 \%$ of the vehicle length down from the nose. Again, the vehicle is trimmed for Mach 8, steady-level flight at an altitude of 85,000 feet. The trimmed state and input variables are given in Table 14. Note that $\psi, x_{E}$, and $y_{E}$ are not included because they do not contribute to the vehicle dynamics directly.

Table 13. Vehicle Mass Properties

\begin{tabular}{cc}
\hline \hline Property & Value \\
\hline$m$ & $9.68 \times 10^{4} \mathrm{~kg}$ \\
$I_{x x}$ & $8.03 \times 10^{5} \mathrm{~kg} \cdot \mathrm{m}^{2}$ \\
$I_{y y}$ & $4.02 \times 10^{6} \mathrm{~kg} \cdot \mathrm{m}^{2}$ \\
$I_{z z}$ & $6.02 \times 10^{6} \mathrm{~kg} \cdot \mathrm{m}^{2}$ \\
\hline \hline
\end{tabular}

Table 14. 3D Steady-Level Flight Trim

\begin{tabular}{cc}
\hline \hline State & Value \\
\hline$V$ & $7847 \mathrm{ft} / \mathrm{s}$ \\
$h\left(-z_{E}\right)$ & $85,000 \mathrm{ft}$ \\
$\alpha$ & $0.942 \mathrm{deg}$ \\
$\beta$ & $0 \mathrm{deg}$ \\
$\theta$ & $0.942 \mathrm{deg}$ \\
$\phi$ & $0 \mathrm{deg}$ \\
$p$ & $0 \mathrm{deg} / \mathrm{s}$ \\
$q$ & $0 \mathrm{deg} / \mathrm{s}$ \\
$r$ & $0 \mathrm{deg} / \mathrm{s}$ \\
$\delta_{c}$ & $-2.43 \mathrm{deg}$ \\
$\delta_{e}$ & $6.42 \mathrm{deg}$ \\
$\Phi$ & 0.476 \\
\hline \hline
\end{tabular}

The 3D flight dynamics are then linearized about this trim state. The eigenvalues and eigenvectors of the linear system are given in Tables 15 and 16. Sets of eigenvalues are assigned to the classical flight dynamics modes (phugoid, Dutch-roll, etc.). These assignments are chosen qualitatively and may not exactly match the standard behavior of the classical modes.

The fastest flight mode of the vehicle is an unstable short period mode. Additionally, there is a stable phugoid mode that is strongly coupled with the stable altitude mode. The lateral dynamics feature an unstable oscillatory mode (Dutch-roll), a stable exponential mode (roll), and a very slow stable exponential mode (spiral). From these results, it is apparent that stabilizing feedback control will be required to maintain steady-level flight both longitudinally and laterally.

\section{Concluding Remarks}

This paper presents a three-dimensional hypersonic vehicle simulation framework in which controllability aspects of the vehicle can be assessed for configuration tradeoffs as well as to evaluate control designs. In its first implementation, the framework accounts for the six rigid-body degrees of freedom, parametrized 
Table 15. Eigenvalues of Linearized System

\begin{tabular}{cccc}
\hline \hline Eigenvalue & Damping Ratio & Natural Frequency $(\mathrm{rad} / \mathrm{s})$ & Mode \\
\hline 5.46 & -1.00 & 5.46 & Short Period \\
-5.60 & 1.00 & 5.60 & Short Period \\
$-7.34 \times 10^{-6} \pm 0.0392 j$ & $1.87 \times 10^{-4}$ & 0.0392 & Phugoid \\
$-1.31 \times 10^{-3}$ & 1.00 & $1.31 \times 10^{-3}$ & Altitude \\
$5.44 \times 10^{-3} \pm 1.07 j$ & $-5.10 \times 10^{-3}$ & 1.07 & Dutch Roll \\
$-1.32 \times 10^{-3}$ & 1.00 & $1.32 \times 10^{-3}$ & Roll \\
$-1.85 \times 10^{-15}$ & 1.00 & $-1.85 \times 10^{-15}$ & Spiral \\
\hline \hline
\end{tabular}

Table 16. Eigenvectors of Linearized System

\begin{tabular}{ccccc}
\hline \hline State & Short Period & Short Period & Phugoid & Altitude \\
\hline$u$ & $1.90 \times 10^{-2}$ & $1.39 \times 10^{-2}$ & $4.07 \times 10^{-3} \pm 5.92 \times 10^{-5} j$ & $-2.81 \times 10^{-1}$ \\
$v$ & $-2.12 \times 10^{-11}$ & $-2.03 \times 10^{-11}$ & $2.02 \times 10^{-13} \pm 6.80 \times 10^{-13} j$ & $-1.72 \times 10^{-7}$ \\
$w$ & -0.999 & -0.999 & $6.82 \times 10^{-5} \pm 2.76 \times 10^{-6} j$ & $-3.73 \times 10^{-2}$ \\
$p$ & $-1.35 \times 10^{-12}$ & $1.32 \times 10^{-12}$ & $3.81 \times 10^{-15} \pm 7.95 \times 10^{-14} j$ & $-2.11 \times 10^{-8}$ \\
$q$ & $-2.34 \times 10^{-3}$ & $2.28 \times 10^{-3}$ & $6.43 \times 10^{-7} \pm 2.62 \times 10^{-10} j$ & $-1.18 \times 10^{-9}$ \\
$r$ & $2.62 \times 10^{-14}$ & $-2.57 \times 10^{-14}$ & $-8.20 \times 10^{-15} \pm 1.69 \times 10^{-15} j$ & $6.18 \times 10^{-8}$ \\
$\phi$ & $-2.47 \times 10^{-13}$ & $-2.35 \times 10^{-13}$ & $-2.02 \times 10^{-12} \pm 9.33 \times 10^{-14} j$ & $1.53 \times 10^{-5}$ \\
$\theta$ & $-4.29 \times 10^{-4}$ & $-4.08 \times 10^{-4}$ & $3.60 \times 10^{-9} \pm 1.64 \times 10^{-5} j$ & $9.02 \times 10^{-7}$ \\
$h\left(-z_{E}\right)$ & $4.57 \times 10^{-3}$ & $4.35 \times 10^{-3}$ & -1.00 & 0.960 \\
\hline \hline
\end{tabular}

\begin{tabular}{cccc}
\hline \hline State & Dutch Roll & Roll & Spiral \\
\hline$u$ & $3.52 \times 10^{-10} \pm 4.15 \times 10^{-10} j$ & $8.47 \times 10^{-7}$ & $-3.58 \times 10^{-15}$ \\
$v$ & 1.00 & $-1.13 \times 10^{-2}$ & $-7.43 \times 10^{-15}$ \\
$w$ & $-2.19 \times 10^{-8} \pm 1.30 \times 10^{-10} j$ & $1.15 \times 10^{-8}$ & $-4.39 \times 10^{-17}$ \\
$p$ & $-7.74 \times 10^{-7} \pm 1.52 \times 10^{-4} j$ & $-1.39 \times 10^{-3}$ & $-6.70 \times 10^{-5}$ \\
$q$ & $-1.44 \times 10^{-12} \pm 9.76 \times 10^{-12} j$ & $3.48 \times 10^{-15}$ & $1.48 \times 10^{-19}$ \\
$r$ & $2.26 \times 10^{-6} \pm 4.43 \times 10^{-4} j$ & $4.05 \times 10^{3}$ & $4.07 \times 10^{-3}$ \\
$\phi$ & $1.35 \times 10^{-4} \pm 1.38 \times 10^{-6} j$ & 0.999 & 1.00 \\
$\theta$ & $-9.16 \times 10^{-12} \pm 1.31 \times 10^{-12} j$ & $-2.63 \times 10^{-12}$ & $-7.82 \times 10^{-19}$ \\
$h\left(-z_{E}\right)$ & $-3.05 \times 10^{-9} \pm 4.80 \times 10^{-11} j$ & $-2.92 \times 10^{-6}$ & $2.44 \times 10^{-13}$ \\
\hline \hline
\end{tabular}


geometry definition with multiple control surfaces, 3D steady aerodynamics, and a simplified 1D scramjet model. The new aerodynamic formulation showed good agreement with the CFD results for the top and bottom surfaces of the vehicle for most of the trials conducted. Pressure in the center region of the surfaces was shown to be effectively found by using the two-dimensional shock/expansion equations, while the Mach angle provided the boundary for the edge effects. The side formulation showed good agreement with the CFD data on the shock side for all trials, though the errors on the shadow side were higher in some instances. The inclusion of these formulations into the six-degree-of-freedom hypersonic vehicle model provides more insight into vehicle flight dynamics than to simply use two-dimensional shock/expansion values over the entire surface. When implemented in a flight dynamics framework, the aerodynamic and propulsion models yielded qualitatively similar longitudinal stability properties as found in existing models. Additionally, the lateral flight dynamics were analyzed and shown to be unstable. The analysis tools developed for this study will support the design of hypersonic flight control systems, as well as to perform 3D vehicle configuration studies. Future enhancements include an improved 3D scramjet model; unsteady, viscous aerodynamics effects along with body/control surface shock interactions; and elastic structures under thermal effects.

\section{Acknowledgements}

This research is funded by NASA award NNX08AB32A (Don Soloway as Associate Principal Investigator and Peter Ouzts as technical monitor) and by the Air Force Research Laboratory/Air Vehicles Directorate grant FA 8650-07-2-3744 for the Michigan/AFRL Collaborative Center in Control Sciences (David Doman as technical monitor).

\section{References}

\footnotetext{
${ }^{1}$ Bolender, M. A. and Doman, D. B., "Nonlinear Longitudinal Dynamical Model of an Air-Breathing Hypersonic Vehicle," Journal of Spacecraft and Rockets, Vol. 44, No. 2, 2007, pp. 374-387.

${ }^{2}$ Oppenheimer, M. W., Skujins, T., Bolender, M. A., and Doman, D. B., "A Flexible Hypersonic Vehicle Model Developed with Piston Theory," Proceedings of the 2007 Atmospheric Flight Mechanics Conference and Exhibit, AIAA Paper No. 20076396, August 2007.

${ }^{3}$ Skujins, T., Cesnik, C. E., Oppenheimer, M. W., and Doman, D. B., "Applicability of an Analytical Shock/Expansion Solution to the Elevon Control Effectiveness for a 2-D Hypersonic Vehicle Configuration," Proceedings of the 2008 Atmospheric Flight Mechanics Conference and Exhibit, AIAA Paper No. 2008-6384, August 2008.

${ }^{4}$ Chavez, F. R. and Schmidt, D. K., "An Integrated Analytical Aeropropulsive/Aeroelastic Model for the Dynamic Analysis of Hypersonic Vehicles," Proceedings of the 1992 Atmospheric Flight Mechanics Conference, AIAA Paper No. 1992-4567, August 1992.

${ }^{5}$ Mirmirani, M., Wu, C., Clark, A., Choi, S., and Colgren, R., "Modeling for Control of a Generic Airbreathing Hypersonic Vehicle," Proceedings of the 2005 Guidance, Navigation and Control Conference, AIAA Paper No. 2005-6256, August 2005.

${ }^{6}$ Torrez, S. M., Driscoll, J. F., Dalle, D. J., and Micka, D. J., "Scramjet Engine Model MASIV: Role of Mixing, Chemistry and Wave Interaction," Proceedings of the 2009 AIAA Joint Propulsion Conference and Exhibit, AIAA Paper No. 2009-4939, August 2009.

${ }^{7}$ Torrez, S. M., Driscoll, J. F., Dalle, D. J., and Fotia, M. L., "Hypersonic Vehicle Thrust Sensitivity to Angle of Attack and Mach Number," Proceedings of the 2009 AIAA Atmospheric Flight Mechanics Conference, AIAA Paper No. 2009-6152, August 2009.

${ }^{8}$ Stengel, R. F., Flight Dynamics, Princeton University Press, Princeton, NJ, 2004.

${ }^{9}$ Starkey, R. P. and Lewis, M. J., "A Shock-Expansion Method for Determining Surface Properties On Irregular Geometries," Proceedings of the 40th Aerospace Sciences Meeting and Exhibit, AIAA Paper No. A02-14357, January 2002.

${ }^{10}$ Nastase, A. and Weisensee, U., "Prediction of Pressure Distribution $C_{p}$ on a Long Rectangular Wing in TransonicSupersonic Flow," Zeitschrift fur angewandte Mathematik und Mechanik, Vol. 64, No. 4, 1984, pp. T212-T214.

${ }^{11}$ Nastase, A. and Honermann, A., "The Three-Dimensional Representation of the Pressure Distribution on Wedged Delta Wings with Supersonic Leading Edges in Supersonic-Hypersonic Flow," Zeitschrift fur angewandte Mathematik und Mechanik, Vol. 73, No. 6, 1993, pp. T603-T607.

${ }^{12}$ Sims, J. L., "Tables for supersonic flow around right circular cones at zero angle of attack," Tech. Rep. NASA SP -3004, Office of Scientific and Technical Information, NASA, 1964.

${ }^{13}$ Anon., "CFD++ User Manual," Tech. rep., Metacomp Technologies, Inc., Agoura Hills, California, 2006.

${ }^{14}$ Anon., "Documentation for ANSYS ICEM CFD/AI*Environment 11.0," Tech. rep., ANSYS, Canonsburg, Pennsylvania, 2007.

${ }^{15}$ Anon., "Gambit 2.3 User's Guide," Tech. rep., Fluent, Inc., Lebanon, New Hampshire, 2006.
} 